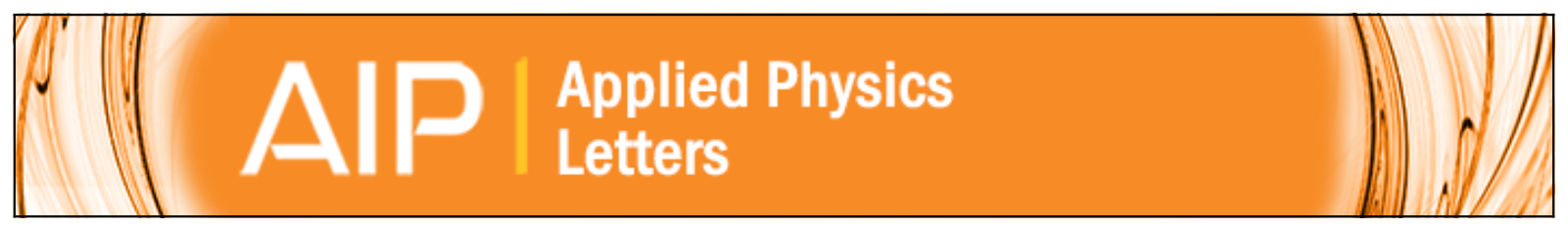

\title{
Amplified spontaneous emission from a conjugated polymer undergone a high- temperature lithography cycle
}

Dario Pisignano, Elisa Mele, Luana Persano, Giovanni Paladini, and Roberto Cingolani

Citation: Applied Physics Letters 86, 261104 (2005); doi: 10.1063/1.1946189

View online: http://dx.doi.org/10.1063/1.1946189

View Table of Contents: http://scitation.aip.org/content/aip/journal/apl/86/26?ver=pdfcov

Published by the AIP Publishing

\section{Articles you may be interested in}

Enhancement of optical gain and amplified spontaneous emission due to waveguide geometry in the conjugated polymer poly[2-methoxy-5-(2'-ethylhexyloxy)-p-phenylene vinylene]

Appl. Phys. Lett. 102, 073303 (2013); 10.1063/1.4793422

Low threshold amplified spontaneous emission from dye-doped DNA biopolymer

J. Appl. Phys. 111, 113107 (2012); 10.1063/1.4728218

Comment on "Gain coefficient method for amplified spontaneous emission in thin waveguided film of a conjugated polymer" [Appl. Phys. Lett.93, 163307 (2008)]

Appl. Phys. Lett. 94, 106101 (2009); 10.1063/1.3097382

Amplified spontaneous emission from cyano substituted oligo( $p$-phenylene vinylene) single crystal with very high photoluminescent efficiency

Appl. Phys. Lett. 90, 141110 (2007); 10.1063/1.2720298

Highly directional stimulated emission from a polymer waveguide

J. Appl. Phys. 84, 5445 (1998); 10.1063/1.368858 


\title{
Amplified spontaneous emission from a conjugated polymer undergone a high-temperature lithography cycle
}

\author{
Dario Pisignano, ${ }^{\text {a) }}$ Elisa Mele, Luana Persano, Giovanni Paladini, and Roberto Cingolani \\ NNL, National Nanotechnology Laboratory of Istituto Nazionale di Fisica della Materia (INFM), \\ and Agilent Technologies joint lab clo Dipartimento di Ingegneria dell'Innovazione, Università di Lecce, \\ via Arnesano, I-73100 Lecce, Italy
}

(Received 17 January 2005; accepted 10 May 2005; published online 20 June 2005)

\begin{abstract}
We investigated the amplified spontaneous emission (ASE) and waveguiding properties of a conjugated polymer film after a heating cycle typical of soft lithography procedures. We found a maximum gain coefficient of $8 \mathrm{~cm}^{-1}$, with excitation density and length thresholds for ASE-induced line narrowing of $200 \mu \mathrm{J} \mathrm{cm}{ }^{-2}$ and $0.9 \mathrm{~mm}$, respectively. Importantly, we found a loss coefficient of the organic slab as low as $5.4 \mathrm{~cm}^{-1}$, which is among the best results reported for organic waveguide amplifiers. These results are important in view of the application of polymer films as active layers for laser devices realized by patterning with high-temperature mechanical lithographies. (C) 2005 American Institute of Physics. [DOI: 10.1063/1.1946189]
\end{abstract}

Finding and characterizing gaining conjugated compounds is a key issue for the fabrication of organic-based solid-state lasers. Pump-probe experiments, measuring the differential transmission of a probe pulse under optical pumping, are the most direct way to determine the gain spectral region and cross section. ${ }^{1-3}$ Another useful method for discriminating if a conjugated material exhibits optical gain is looking for amplified spontaneous emission (ASE). ${ }^{4}$ In the last years, a lot of attention has indeed been paid to ASE in conjugated polymers $^{5-7}$ and oligomers, ${ }^{8,9}$ aimed at employing these novel low-cost compounds as active media of solid-state resonators, and particularly distributed-feedback ${ }^{10}$ and photonic crystal $^{11,12}$ lasing devices.

The feedback in such cavities occurs due to the Bragg diffraction induced by a periodic corrugation, continuously reflecting a part of the wave propagating in the forward direction of the medium. Besides nonmorphological, ${ }^{13,14}$ or photoinduced surface patterning, ${ }^{15,16}$ the most promising lithographic approaches to fabricate wavelength-scale periodic structures in organic materials are various soft ${ }^{17,18}$ and nanoimprinting ${ }^{19,20}$ methods. Many of them exploit the glass transition of amorphous organics, namely they work by decreasing the viscosity driving the materials above their glasstransition temperature, $T_{g}$, upon placing the films in contact with a previously fabricated mold. However, thermal cycles may cause damage to active conjugated molecules, for instance through the heating-induced carbonyl $(\mathrm{C}=\mathrm{O})$ substitution of the vinyl group in poly-(phenylenevinylene) compounds. ${ }^{21}$ For this reason, the functionality of gaining conjugated molecules after thermal cycles has to be fully assessed. In this letter, we demonstrate that a conjugated polymer film exhibits a very effective amplified spontaneous emission (with a measured threshold excitation density for line narrowing of about $200 \mu \mathrm{J} \mathrm{cm}^{-2}$ and a threshold length of less than $1 \mathrm{~mm}$ ) and waveguiding properties (losses of about $5.4 \mathrm{~cm}^{-1}$ ) even after a heating cycle, typical of a lithographic process. These results strongly support the use of a high-temperature soft lithographic process for realizing distributed feedback laser resonators.

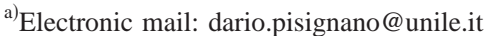

As a prototype polymer, we chose the poly[(9,9hexylfluorenyl-2,7-ditl)-alt-co-(9-ethyl-3,6-carbazole)]

[(PFC) American Dye Source, Inc., Canada]-molecular structure in Fig. 1(a). PFC films were cast by spin coating from $3 \times 10^{-3} \mathrm{M}$ dichloromethane solutions on Corning glass substrates $(n=1.46)$. With a spin-coating speed of $2000 \mathrm{rpm}$, we obtained neat films of thicknesses about $430 \mathrm{~nm}$, measured by a profilometer. The films were then heated at $170{ }^{\circ} \mathrm{C}$ for 5 min under a nitrogen atmosphere. Such values of temperature and heating times are characteristic of a typical nanoimprinting or molding process. The third harmonic $(\lambda$ $=355 \mathrm{~nm}$ ) of a $3 \mathrm{~ns} Q$-switched Nd:YAG laser (SpectraPhysics, repetition rate of $10 \mathrm{~Hz}$ ) was employed as the excitation source. The pump beam was focused on the sample by a cylindrical lens providing a rectangular excitation stripe of width $500 \mu \mathrm{m}$. The sample emission at room temperature was collected from the sample edge, dispersed by a mono-
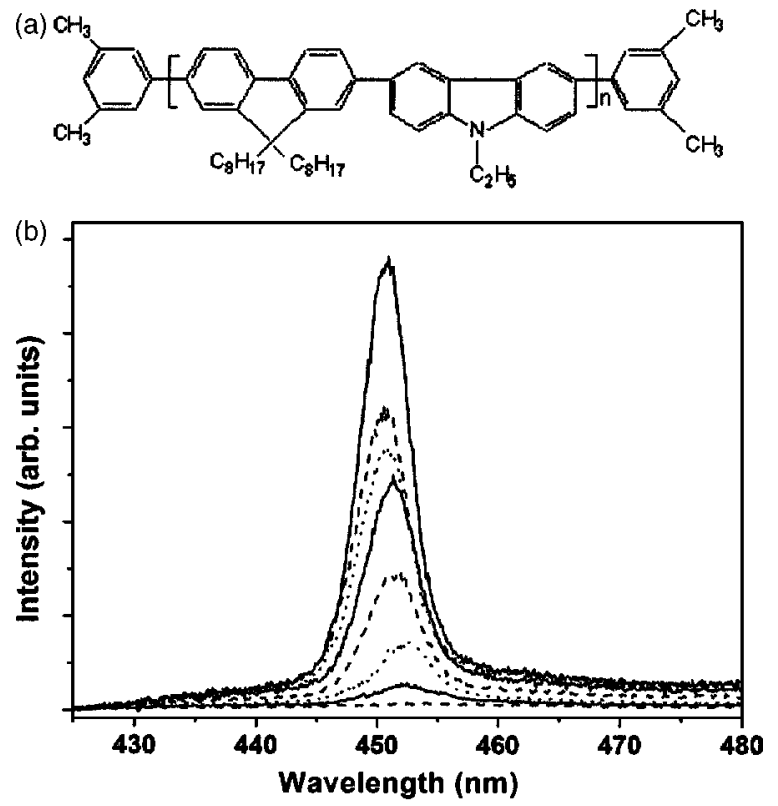

FIG. 1. (a) Molecular structure of PFC. (b) Emission spectra from PFC films under different excitation fluences (from bottom to top: 0.2, 0.4, 0.9, 2.5, $4.2,5.1,6.2$, and $10.1 \mathrm{~mJ} \mathrm{~cm}^{-2}$ ). 


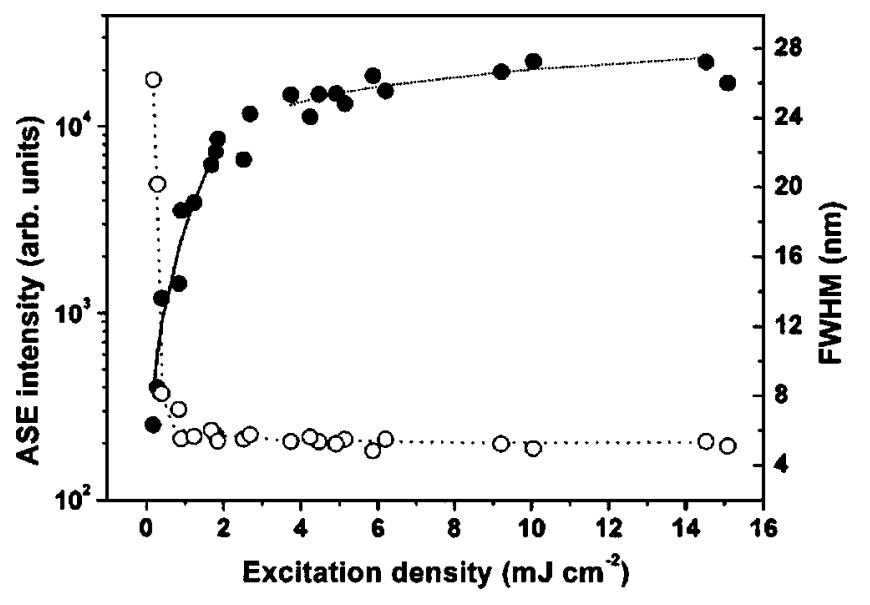

FIG. 2. Dependence of the ASE integrated emitted intensity (solid circles, left scale) and spectral width [full width at half maximum (FWHM), open circles, right scale] on the pump energy density. The continuous line is the best fit to the data up to $1.8 \mathrm{~mJ} \mathrm{~cm}^{-2}$ by an exponential-like ASE model. The dotted line is the best fit of the data between $3.7 \mathrm{~mJ} \mathrm{~cm}^{-2}$ and $14.5 \mathrm{~mJ} \mathrm{~cm}^{-2}$ to the square root behavior predicted by the bimolecular annihilation model. The dashed curve interpolating the FWHM data is a guide for the eyes only.

chromator, and detected by a Si charge coupled device. The measurements were carried out under a vacuum $\left(10^{-6}\right.$ mbar $)$ to inhibit photo-oxidation.

The photoluminescence (PL) spectra, as a function of the excitation density, are displayed in Fig. 1(b). For excitation fluences, $E$, larger than $200 \mu \mathrm{J} \mathrm{cm}^{-2}$, the narrow peak at 454 $\mathrm{nm}$ (roughly corresponding to the $0-1$ vibrational replica of the low-power fluorescence spectrum) becomes more and more evident, resulting in a significant line narrowing, from 26 to $5 \mathrm{~nm}$. This effect is ascribed to ASE assisted by the waveguiding effect due to the different refractive index of substrate, gain medium, and vacuum, respectively. The output intensity shows a rapid (approximately exponential) ${ }^{4}$ increase up to about $2 \mathrm{~mJ} \mathrm{~cm}^{-2}$, then saturating for very large excitation intensities $\left(E>3.5 \mathrm{~mJ} \mathrm{~cm}^{-2}\right)$, due to the presence of nonradiative decay processes, whose contribution increases upon increasing the pump density. Such a behavior, typical of several conjugated molecules, has been mainly attributed to nonlinear effects, such as the bimolecular exciton annihilation, which is due to the high density of excitons formed under intense photon fluxes. ${ }^{22}$ This model is indeed consistent with the square-root dependence of $E$ for large pump densities (dotted curve fitting the data in Fig. 2). ${ }^{22,23}$

In order to assess in depth the gain performances of PFC after the thermal cycle, we carried out measurements by the variable stripe length method, which provides the optical gain spectrum, $g(\lambda)$, of PFC through the relation between the emitted intensity, $I$, and the excitation length, $\ell:{ }^{24} I(\lambda)$ $=I_{0}(\lambda)\{\exp [g(\lambda) \ell]-1\}$. The PL spectra collected with different excitation stripes are reported in Fig. 3, showing that the line narrowing is enhanced by longer pump lengths (inset of Fig. 4). The gain spectrum shows a well-defined peak, centered at about $450 \mathrm{~nm}$ with a spectral width of $7 \mathrm{~nm}$ and a maximum of about $8 \mathrm{~cm}^{-1}$. By the value of the maximum optical gain, one can extract the threshold length, $\ell_{\mathrm{th}}$, needed to observe ASE, via the relation: $\exp \left[g(\lambda) \ell_{\mathrm{th}}\right]-1=1$, i.e., $\ell_{\mathrm{th}}=\ln 2 / g(\lambda)$. We obtain for our PFC: $\ell_{\mathrm{th}}=(0.9 \pm 0.1) \mathrm{mm}$, in perfect agreement with the experimental finding about line narrowing (inset of Fig. 4). Moreover, one can estimate the stimulated emission cross section:

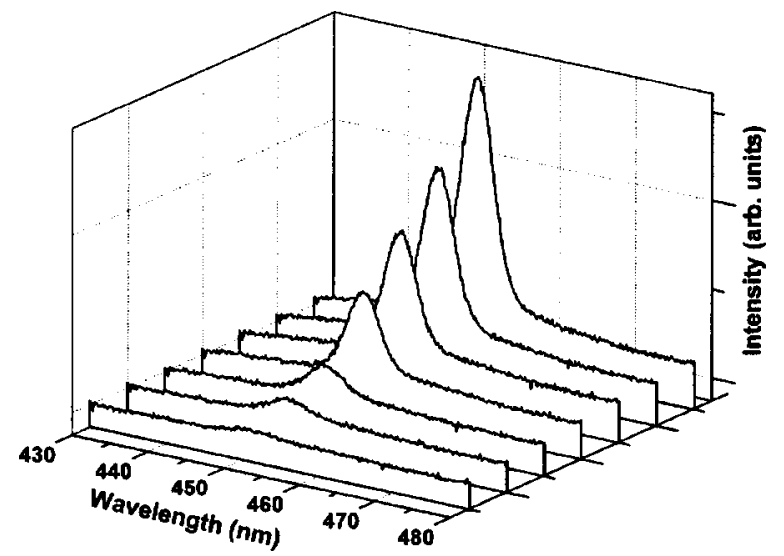

FIG. 3. Emission spectra by different excitation length: 1.07 (a), 2.39 (b), 3.71 (c), 5.03 (d), 6.78 (e), 7.66 (f), and $8.54 \mathrm{~mm}$ (g).

$$
\sigma_{g}=\frac{g}{N}
$$

where $N$ is the excitation density. We obtain $\sigma_{g}=4.9$ $\times 10^{-19} \mathrm{~cm}^{2}$ (with $N=1.6 \times 10^{19} \mathrm{~cm}^{-3}$ ), which is significantly larger than the value obtained by fitting the ASE output behavior, although still one order of magnitude lower than the typical values of some prototype conjugated oligomers and polymers. ${ }^{25-27}$ However, this calculation assumes unity quantum efficiency for the emitting species (namely, it is calculated by assuming $N$ equal to the number of absorbed photons per unit volume), so it underestimates the actual value of the gain cross section. Further study is currently in progress in our laboratory, aiming to assess the performance of a wide number of conjugated polymers upon thermal cycles, in terms of achievable optical gain.

Finally, we underline that a crucial role within organic optoelectronic devices, and especially laser resonators and optical amplifiers, is played. by the waveguiding capability of the active films. For this reason, we were particularly interested in determining the waveguide performances of our organic slab upon heating. The guiding losses were determined by fixing the excitation length $(\ell=4 \mathrm{~mm})$, and by moving the pump stripe away from the emission edge of the sample, thus increasing the length $(d)$ of the unpumped region. $^{28,29}$ The output intensity decreases roughly exponentially upon increasing $d$, suggesting the predominance of ab-

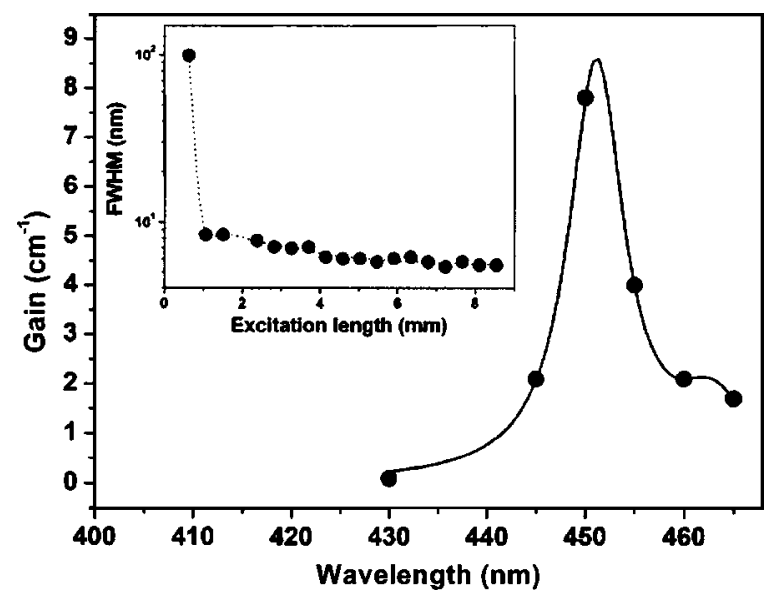

FIG. 4 Optical gain spectrum for an excitation density of $390 \mu \mathrm{J} \mathrm{cm}^{-2}$. The continuous line is a guide for the eyes. Inset: FWHM vs excitation length. 


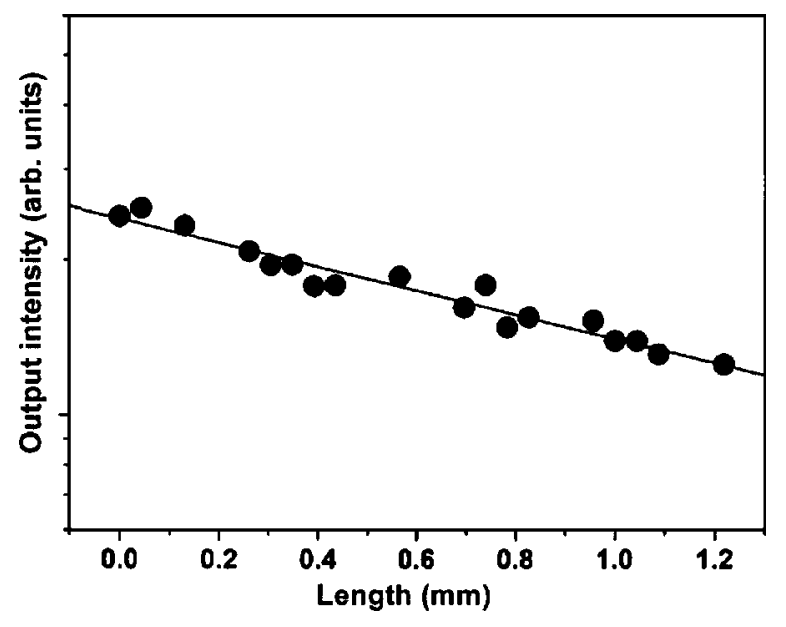

FIG. 5. Dependence of the ASE output from the slab edge on the length $(d)$ of the nonexcited region between the pump stripe and the edge of the sample. The dotted line is the best fit of the data to the equation $I=I_{0} \exp (-\alpha d)$.

sorption losses (Fig. 5). The measured loss coefficient of the PFC waveguide is $\alpha=(5.4 \pm 0.3) \mathrm{cm}^{-1}$, which is one order of magnitude lower than that found in the poly-[2methoxy-5-(2'-ethyl-hexiloxy)-1,4-phenylenevinylene], ${ }^{28}$ and of the same order of the best results reported for conjugated polyfluorenes. ${ }^{29}$ The heating procedure, driving the polymer above the glass transition and thus inducing a structural rearrangement of the amorphous film, may undoubtedly contribute to improving the waveguiding capability of the organic slab. The resulting smoothing of the polymer surface can also contribute to reduce scattering losses.

In conclusion, we have presented a study of the ASE emission from a conjugated polymer undergone a typical high-temperature lithographic cycle. The determined excitation density and length thresholds for ASE-induced line narrowing (200 $\mu \mathrm{J} \mathrm{cm}^{-2}$ and $0.9 \mathrm{~mm}$, respectively), and the very low coefficient of waveguide losses $\left(5.4 \mathrm{~cm}^{-1}\right)$, all indicate that the material is fully functional as a possible active medium for laser resonators. In fact, organic-based distributed feedback lasers ${ }^{1420}$ can be realized by compounds with gain coefficients $\left(\cong 10 \mathrm{~cm}^{-1}\right)$ and guide losses in the same range as those here found for PFC. Since patterning conjugated materials for realizing distributed feedback and photonic crystal structures is strategic for achieving electrically injected organic solid-state lasers (the coupling between the electronic excitations and photons being directly affected by the output optical mode density), and since high-temperature soft lithography and nanoimprinting are the best approaches to pattern organic molecules, this result is an important step forward toward electrically pumped organic lasing.

${ }^{1}$ W. Graupner, G. Leising, G. Lanzani, M. Nisoli, S. De Silvestri, and U. Scherf, Phys. Rev. Lett. 76, 847 (1996).

${ }^{2}$ S. V. Frolov, Z. V. Vardeny, and K. Yoshino, Phys. Rev. B 57, 9141 (1998).

${ }^{3}$ M. Nisoli, S. Stagira, M. Zavelani-Rossi, S. De Silvestri, P. Mataloni, and C. Zenz, Phys. Rev. B 59, 11328 (1999).

${ }^{4}$ O. Svelto, Principles of Lasers (Plenum, New York, 1989).

${ }^{5}$ F. Hide, M. A. Díaz-García, B. J. Schwartz, M. R. Andersson, Q. Pei, and A. J. Heeger, Science 273, 1833 (1996).

${ }^{6}$ G. J. Denton, N. Tessler, M. A. Stevens, and R. H. Friend, Adv. Mater. (Weinheim, Ger.) 9, 547 (1997).

${ }^{7}$ R. Xia, G. Heliotis, and D. D. C. Bradley, Appl. Phys. Lett. 82, 3599 (2003).

${ }^{8}$ N. Johansson, J. Salbeck, J. Bauer, F. Weissörtel, P. Bröms, A. Andersson, and W. R. Salaneck, Adv. Mater. (Weinheim, Ger.) 10, 1136 (1998).

${ }^{9}$ D. Pisignano, M. Anni, G. Gigli, R. Cingolani, M. Zavelani-Rossi, G. Lanzani, G. Barbarella, and L. Favaretto, Appl. Phys. Lett. 81, 3534 (2002).

${ }^{10}$ H. Kogelnik and C. V. Shank, Appl. Phys. Lett. 18, 152 (1971).

${ }^{11}$ J. D. Joannopoulos, R. D. Meade, and J. N. Winn, Photonic CrystalsMolding the Flow of Light (Princeton University Press, Princeton, New Jersey, 1995).

${ }^{12}$ M. Meier, A. Mekis, A. Dodabalapur, A. Timko, R. E. Slusher, J. D. Joannopoulos, and O. Nalamasu, Appl. Phys. Lett. 74, 7 (1999).

${ }^{13}$ M. Nagawa, M. Ichikawa, T. Koyama, H. Shirai, Y. Taniguchi, A. Hongo, S. Tsuji, and Y. Nakano, Appl. Phys. Lett. 77, 2641 (2000).

${ }^{14}$ N. Tsutsumi, T. Kawahira, and W. Sakai, Appl. Phys. Lett. 83, 2533 (2003).

${ }^{15}$ V. Dumarcher, L. Rocha, C. Denis, C. Fiorini, J.-M. Nunzi, F. Sobel, B. Sahraoui, and D. Gindre, J. Opt. A, Pure Appl. Opt. 2, 279 (2000).

${ }^{16} \mathrm{~T}$. Maillou, J. L. Moigne, V. Dumarcher, L. Rocha, B. Geffroy, and J.-M. Nunzi, Adv. Mater. (Weinheim, Ger.) 14, 1297 (2002).

${ }^{17}$ K. Y. Suh, Y. S. Kim, and Hong H. Lee, Adv. Mater. (Weinheim, Ger.) 13, 1386 (2001).

${ }^{18}$ D. Pisignano, L. Persano, G. Gigli, R. Cingolani, F. Babudri, G. M. Farinola, and F. Naso, Appl. Phys. Lett. 84, 1365 (2004).

${ }^{19}$ S. Y. Chou, P. R. Krauss, and P. J. Renstrom, Science 272, 85 (1996).

${ }^{20}$ D. Pisignano, L. Persano, P. Visconti, R. Cingolani, G. Gigli, G. Barbarella, and L. Favaretto, Appl. Phys. Lett. 83, 2545 (2003).

${ }^{21}$ M. Yan, L. J. Rothberg, F. Papadimitrakopoulos, M. E. Galvin, and T. M. Miller, Phys. Rev. Lett. 73, 744 (1994).

${ }^{22}$ E. S. Maniloff, V. I. Klimov, and D. W. McBranch, Phys. Rev. B 56, 1876 (1997).

${ }^{23}$ G. J. Denton, N. Tessler, M. A. Stevens, and R. H. Friend, Adv. Mater. (Weinheim, Ger.) 9, 547 (1997).

${ }^{24}$ K. L. Shaklee, R. E. Nahory, and R. F. Leheny, J. Lumin. 7, 284 (1973).

${ }^{25}$ E. S. Maniloff, V. I. Klimov, and D. W. McBranch, Phys. Rev. B 56, 1876 (1997).

${ }^{26}$ B. Schweitzer, G. Wengmann, H. Giessen, D. Hertel, H. Bassler, and R. F. Mahrt, Appl. Phys. Lett. 72, 2933 (1998).

${ }^{27}$ M. Anni, G. Gigli, R. Cingolani, M. Zavelani-Rossi, C. Gadermaier, G. Lanzani, G. Barbarella, and L. Favaretto, Appl. Phys. Lett. 78, 2679 (2001).

${ }^{28}$ M. D. McGehee, R. Gupta, S. Veenstra, E. K. Miller, M. A. Diaz-Garcia, and A. J. Heeger, Phys. Rev. B 58, 7035 (1998).

${ }^{29}$ G. Heliotis, D. D. C. Bradley, G. A. Turnbull, and I. D. W. Samuel, Appl. Phys. Lett. 81, 415 (2002). 